\title{
Student Future Prediction System Under Filtering Mechanism
}

\author{
L.K. Joshila Grace
}

\begin{abstract}
Information is progressively being utilized to improve regular day to day existence less demanding and. Applications, for example, holding up time estimation, traffic expectation, and stopping look are genuine instances of how information from various sources can be utilized to encourage our day by day life.In this period of computerization, instruction has additionally patched up itself and isn't constrained to old address strategy. The ordinary mission is on to discover better approaches to make it increasingly successful and effective for understudies. These days, loads of information is gathered in instructive databases, yet it remains unutilized. So as to get required advantages from such major information, amazing assets are required. Information mining is a developing integral asset for investigation and forecast. In this investigation, we consider an under-used information source: college ID cards. Such cards are utilized on numerous grounds to buy nourishment, enable access to various territories, and even gauge participation in classes. In this article, we use information from our college to investigate use of the college wellness focus and fabricate an indicator for future visit volume. The work makes a few commitments: it exhibits the extravagance of the information source, demonstrates how the information can be utilized to improve understudy administrations, finds fascinating patterns and conduct, and fills in as a contextual analysis outlining the whole information science process. One objective of this article is to show the information science process. As far as information accumulation, two arrangements of information-timestamp information from card swipes at the Student Recreation Centre (SRC) and client profile information-were gathered from our Management of University. The gathered information was cleaned and further prepared. At that point, exploratory information investigation was performed to find fascinating examples. General understudy practice patterns were found from the timestamp dataset. These incorporate yearly/month to month/every day frequencies of understudy visits to the SRC and pinnacle hours amid multi day.
\end{abstract}

Keyword - Student Recreation Centre (SRC), Identity Card (ID), Data Mining

\section{INTRODUCTION}

In a decade ago, the quantity of advanced education colleges/foundations have multiplied manifolds. Extensive quantities of alumni/post graduates are created by them consistently. Colleges/Institutes may pursue best of the instructional methods; yet at the same time they face the issue of dropout understudies, low achievers and jobless understudies [14]. Understanding and dissecting the components for poor execution is an intricate and relentless procedure covered up in over a wide span of time data congregated from scholastic execution and understudies' conduct. Amazing assets are required to break down and

Revised Manuscript Received on July 18, 2019.

L.K. Joshila Grace Professor, School of Computing,

Sathyabama Institute of Science and Technology, Chennai, Tamilnadu, India.(Email: joshilagracejebin@gmail.com) anticipate the execution of understudies deductively. The colleges/organizations gather a gigantic measure of understudies' information, yet this information remains unutilized and does not help in any choices or approach making to improve the execution of understudies $[12,13]$. The Student Recreation Centre (SRC) is among the most much of the time visited grounds offices in the college. Section to the SRC is checked through the utilization of an official college ID card which clients use upon their passage. Information collected from these card- swipe exercises are rich and can be utilized to increase profitable experiences into grounds life and exercise practices. Notwithstanding, little consideration has been paid in the past to this potential. One of the more extensive objectives of this is work is to illustrate the most effective method to saddle this potential by means of watchful examination and to goad extra investigations on grounds centred information disclosure. The SRC is a well known spot to visit on grounds, and alongside the notoriety, two unmistakable kinds of necessities emerge normally. From the SRC directors' perspective, knowing the utilization example of the offices is essential so as to give tasteful understudy administrations. From the understudies' perspective, knowing future visit volumes is vital to probably dodge the SRC when it is generally swarmed. This work goes for tending to these twin needs at the same time. So to utilize the data for better career predictions of the students we created three diverse expectation models to anticipate future visit volumes at the SRC and examined which of the models plays out the best for our dataset. We assembled an electronic application to show expectation results, which has helped SRC directors in improving the nature of understudy administrations.

\section{RELATED WORK ANALYSIS}

The past and ebb and flow inquire about reports about student future prediction with the help of the activities he is participating and his interests in the university using filtering system have been contemplated. Every one of these reports are taken as a base for this paper. [1] It is seen that there are explicit abilities that graduates need to have so as to end up employable and that these abilities are past general scholastic instruction. Characteristics like passionate insight, self-administration, and Work and beneficial experience are likewise critical elements for Employability Development Profile (EDP). Bosses additionally place the most astounding significance on delicate aptitudes and the most minimal significance on scholarly notoriety. In this manner,

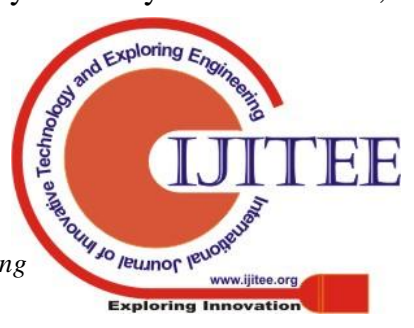


saw employability is fixing to capabilities and airs, as opposed to just on scholastic capability. Critical contrasts are found among scholastic and boss expertise evaluations recommending noticeable expertise hole among the scholarly community and corporate. [2] The consequence of this investigation demonstrates that information mining strategies abilities given viable improving instruments to investigation understudy execution. In this paper, information mining is used to investigate course assessment surveys. Here, the most critical factors that different "acceptable" and "not palatable" understudy exhibitions and there shortcoming's specifically subject or field.Tree-based techniques characterize occasions by arranging the examples down the tree from the root to some leaf hub, which gives the characterization of a specific example. [3] In this paper, three managed information mining calculations were connected on the preoperative appraisal information to anticipate achievement in a course (either passed or fizzled) and the execution of the learning strategies were assessed based on their prescient precision, simplicity of learning also, easy to use attributes. The outcomes demonstrate that the Naïve Bayes classifier beats in expectation decision tree and neural system techniques. It has too been demonstrated that a decent classifier show must be both exact and fathomable for teachers. [4] This investigation model will support instructors recognize how or to what degree their understudies perform distinctively so educators can pick suitable instructing and educational mediations to improve their educating technique[14,15]. It additionally causes instructors to examine the understudy achievement and disappointment, getting the higher mark in the test likewise is accessible here. This investigation will help to improve the understudy's scholastic execution and recognize poor understudies and give some exceptional consideration regarding improve their imprints and limit the level of the disappointment proportion in the tests. [5] Current devices for mining information from a particular course might be valuable as it were to its engineers. There are no broad instruments or re-utilizing devices or procedures that can be connected to any instructive framework. In this way, an institutionalization of information, and the pre preparing, finding and post handling assignments is required. The information mining apparatus must be coordinated into the e-learning condition as another creator apparatus. All information mining errands (pre preparing, information mining and post handling) must be completed into a solitary application. Input and results gotten with information mining can be straightforwardly connected to the e-learning condition. [6] It has appeared encouraging hypothetical establishment for a careful limited dimensional nonlinear channel as a helpful calculation for reasonable prognostic issues. It has proposed a definite answer for the prognostic vulnerability portrayal, as a Benes channel, to underscore the utility that such a definite detailing has on the nature of the RUL expectation. We have likewise displayed a Bayesian plan that qualify the hyperbolic procedure demonstrate for application to a rigging bearer plate model issue. While applying an on-line parameter learning plan, the capacity of the framework to catch RUL in long-skyline forecasts is surprisingly encouraging. [7] This paper has explored past examinations on foreseeing understudies execution with different logical techniques. The vast majority of the analysts have utilized aggregate evaluation point normal (CGPA) and inward appraisal as information sets. While for forecast procedures, the arrangement strategy is much of the time utilized in instructive information mining zone. Under the grouping procedures, Neural Network and Decision Tree are the two strategies profoundly utilized by the specialists for anticipating understudies execution. Taking everything into account, the meta-examination on anticipating understudies execution has roused us to complete further research to be connected in our condition. It will push the instructive framework to screen the understudies execution logically. [8] Because of the particular idea of match-related highlights to various games, results crosswise over various investigations in this application can for the most part not be looked at straightforwardly. In spite of the expanding utilization of ML models for sport expectation, increasingly precise models are required. This is expected to the high volumes of wagering on game, and for game supervisors looking for helpful information for displaying future coordinating procedures. Accordingly, ML appears a fitting procedure for game expectation since it creates prescient models that can foresee coordinate results utilizing predefined includes in a chronicled dataset. This article basically investigations some ongoing examination on game expectation that have utilized ANN, and following this, we proposed a game outcome expectation 'SRP-CRISP-DM' structure for the perplexing issue of game outcome forecast.

\section{PROPOSED ALGORITHM \& RESULTS}

The purpose of the project is to explore and visualize patterns of student workout activity in terms of time and demographics. We analyzed and compared the predictive accuracy of a seasonal naive model, an ARIMA model, and a random forest model, and found that the random forest model fits our dataset the best. The visit volume at the SRC for a given time interval was approximately predicted by the random forest model. The deployed webpage has assisted the SRC employees in terms of daily operations such as staff scheduling. We hope this work will serve as a case study illustrating the entire data science process, providing useful insights on how data could be collected, processed, explored, and leveraged to create a user-facing data product. The algorithms used in the proposed system are detailed below.

\section{Naive Bayes}

Naive Thomas Bayes classifiers are a gathering of order calculations bolstered Bayes' Theorem. It isn't one recipe anyway a group of calculations wherever every one of them share an average rule, for example each consolidate of choices being characterized is independent each $\}$ elective The dataset is part into 2 components, specificallyinclude grid and the reaction vector. Highlight network contains every one of the vectors (lines) of dataset inside which each vector comprises of the value of ward choices. Reaction 
vector contains the value of advancement variable (forecast or yield) for each column of highlight lattice. Naive Thomas Bayes Classifiers concede the Bayes Theorem, which depends on contingent likelihood or in basic terms, the probability that an occasion (A) will happen given that another occasion (B) has just occurred. Basically, the hypothesis enables a speculation to be refreshed each time new proof is presented. The condition underneath communicates Bayes Theorem inside the language of likelihood.

$$
\mathrm{P}(\mathrm{A} \mid \mathrm{B})=\frac{\mathrm{P}(\mathrm{B} \mid \mathrm{A}) \mathrm{P}(\mathrm{A})}{\mathrm{P}(\mathrm{B})}
$$

\section{Random Forest}

Random forest is an especially labile machine learning methodology with wide numerous applications and most ordinarily utilized in diagnosing. It should be accustomed model the impact of mercantilism on shopper acquisition, retention, and churn or to predict illness risk and status in patients.Random forest is capable of regression and classification. It will handle an outsized variety of options, and it's useful for estimating that of your variables are vital within the underlying information being modelled. Random forest is hard thing for nearly any prediction draw back (even non-linear ones). It is a trending machine learning strategy (it came out of Bell Labs among the 90s) and can be used for widely for both classification and regression techniques. It belongs to an even bigger class of machine learning. Ensemble learning involves the mixture of the many models to resolve one prediction draw back. It is working by generating the multiple classifiers and also models that learn and also build predictions. Those predictions are then combined into one (mega) prediction that ought to be sensible or higher than the prediction created by anyone classifier.

To do expectation misuse the standard of prepared irregular timberland utilizes this pseudo-code. Takes the take a look at decisions and utilize the standards of each at arbitrary made choice tree to foresee the best outcome and stores the foreseen result (target). Compute the votes in favor of each prognosticated target consider the high casted a ballot anticipated focus because of it's a definitive expectation from the irregular woods rule. To play out the expectation misuse the prepared arbitrary backwoods rule we'd want to pass and take a look at decisions through the standards of each at arbitrarily made trees. Assume suppose we tend to tend to planned 100 arbitrary choice trees to from the irregular woodland. Each irregular timberland will foresee very surprising targets (result) for consistent look of highlight. By utilizing each anticipated target cast a ballot are being determined. Assume the 100 arbitrary choice trees territory unit expectation somewhere in the range of 3 particular targets $\mathrm{x}, \mathrm{y}, \mathrm{z}$ then the votes of $\mathrm{x}$ is only out of 100 irregular choice tree what rate trees forecast is $x$. In like manner for option a couple of targets $(y, z)$. In the event that $\mathrm{x}$ is acquiring high votes. Suppose out of one hundred arbitrary call tree sixty trees are anticipating the objective are $\mathrm{x}$. At that point the last word arbitrary woods restore the $\mathrm{x}$ because of the anticipated target.

\section{Arima}

The Time Series mining capacity gives calculations that depend on various hidden model presumptions with a few parameters. The learning calculations attempt to locate the best model and the best parameter esteems for the given information.

The ARIMA calculation additionally fuses occasional parts. In this way this calculation is additionally alluded to as Seasonal ARIMA (SARIMA). The autoregressive piece of the calculation utilizes weighted past qualities while the moving normal part gauges the recently accepted blunders of the time arrangement. The ARIMA calculation, expect the mistake to be autonomous and indistinguishably disseminated from an ordinary conveyance with zero mean. The essential ARMA show works for stationary time arrangement as it were. Stationary time arrangement contains level with mean and equivalent change for the entire time arrangement. Along these lines the coordinated part makes stationary arrangement by separation.Each structure in an ARIMA display relates to a periodicity or intermittent structure. An occasional structure is an example of information that rehashes all through the information arrangement. Some minor variety in the example is permitted, inside factual points of confinement. Periodicity is estimated by the default time units that were utilized in the preparation information. For instance, if the preparation information gives deals information to every day, the default time unit is one day, and every occasional structure are characterized. Every period that is distinguished by the calculation gets its own structure hub. For instance, in the event that you are breaking down day by day deals information, the model may distinguish occasional structures that speak to weeks.

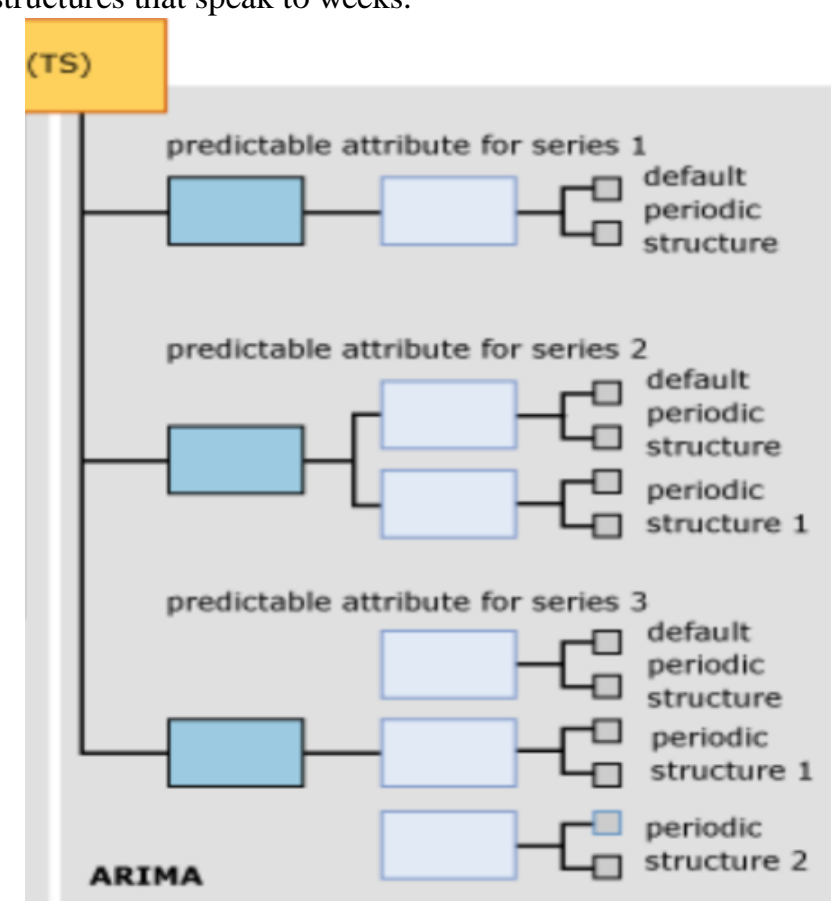

Fig 1 ARIMA

Published By: 
The modules that are indulged are described below for a clear understanding of the system architecture and its way of working. Fig 2 shows the system work flow of proposed model.

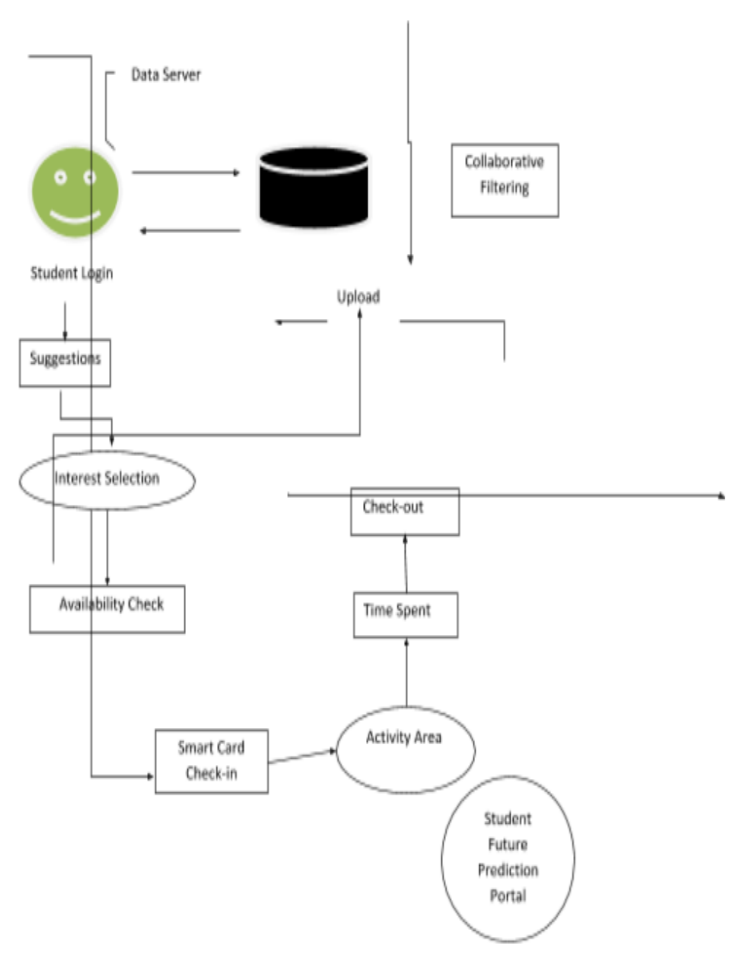

Fig 2 System work flow

\section{Student Id Generation}

Understudies subtleties are filled here alongside the separate reports, id proofs are transferred full information and contact data is gotten from client. Client profile information will be gathered from our University's Data Server and Office of Institutional Research (IR), separately. The gathered information was cleaned and further prepared. At that point, exploratory information examination was performed to find intriguing examples. General understudy practice patterns were found from the timestamp dataset.

\section{Activity Allocation}

Understudies who wish to enter inside any of the office, play region, bottle they should check the seats for different offices and play zones and containers and the demand is sent to database and after that we will acquire the status of the booking. This examination, we consider an under-used information source: college ID cards. Such cards are utilized on numerous grounds to buy nourishment, enable access to various territories, and even gauge participation in classes.

\section{Student Activities Monitoring}

Understudies should look at in with id and check must be made amid whole 4 years of their examinations and every single record are observed and put away inside the database. Information reaped from these card swipe exercises are rich and can be utilized to increase profitable bits of knowledge into grounds life and exercise practices. Be that as it may, little consideration has been paid in the past to this potential. Information collected from these card swipe exercises are rich and can be utilized to increase significant bits of knowledge into grounds life and exercise practices. In any case, little consideration has been paid in the past to this potential. The SRC is a well known spot to visit on grounds, and alongside the prevalence, two unmistakable kinds of necessities emerge normally. From the SRC chiefs' perspective, realizing the utilization example of the offices is critical so as to give attractive understudy administrations. From the understudies' perspective, knowing future visit volumes is vital to almost certainly stay away from the SRC when it is generally swarmed. This work goes for tending to these twin needs at the same time.

\section{Predictions based on Interests}

At last, toward the year's end our territory of intrigue will be created and dependent on that activity notice and recommendations will be made to upgrade our future appropriately. In light of the past gathered information of specific understudy exercises filtration will be connected which helps in creating ideal suggestions.

\section{CONCLUSION}

In this paper, we investigated and envisioned examples of understudy exercise movement as far as time and socioeconomics. We broke down and thought about the prescient exactness of a regular innocent model, an ARIMA display, and an irregular timberland model, and found that the arbitrary woods show it our dataset the best. The visit volume at the SRC for a given time interim was precisely anticipated by the irregular woodland show. The sent website page has helped the SRC representatives interms of everyday activities, for example, staff booking. We trust this work will fill in as a contextual investigation representing the whole information science process, giving valuable bits of knowledge on how information could be gathered, prepared, investigated, and utilized to make a client confronting information item.

\section{REFERENCES}

1. PoojaThakar Assistant Professor VIPS, GGSIPU Delhi, India Anil Mehta, Ph.D Professor University of Rajasthan Jaipur, India Manisha, Ph.D Associate Professor Banasthali University Jaipur, India "Performance Analysis and Prediction in Educational Data Mining: A Research Travelogue", International Journal of Computer Applications, Volume 110 - No. 15, January 2015. Pp. 60-68.

2. Ankita A Nichat, Dr.Anjali B Raut M.E Student, Department of Computer Science \& Engineering, HVPM's College of Engineering \& Technology, Amravati, India HOD, Department of Computer Science \& Engineering, HVPM's College of Engineering \& Technology, Amravati, India "Predicting and Analysis of Student Performance Using Decision Tree Technique", International Journal of Innovative Research in Computer and Communication Engineering, Vol. 5, Issue 4, April 2017. Pp. 7319-7328.

3. EdinOsmanbegović *, MirzaSuljić ** "Data Mining Approach For Predicting Student Performance", Journal of Economics and Business, Vol. X, Issue 1, May 
2012. Pp. 3-12.

4. Dr.RSenthil Kumar1, Jithin Kumar.K.P2 1,2Department of Computer Science, Amrita School of Arts and Sciences, Amrita VishwaVidyapeetham, Mysuru Campus Mysuru, India "Analysis Of Student Performance Based on Classification And Mapreduce Approach In Bigdata", International Journal of Pure and Applied Mathematics, Volume 118 No. 14 2018, 141-148.

5. C. Romero *, S. Ventura Department of Computer Sciences, University of Cordoba, Cordoba, Spain "Educational data mining: A survey from 1995 to 2005", Expert Systems with Applications 33 (2007) 135-146.

6. Jonathan A. DeCastro ${ }^{1}$, Liang Tang ${ }^{1}$, Kenneth A. Loparo ${ }^{2}$, Kai Goebel ${ }^{3}$, George Vachtsevanos ${ }^{4},{ }_{1}^{1}$ Impact Technologies, LLC, Rochester, NY 14623, USA \{jonathan.decastro; liang.tang\}@impact-tek.com ${ }^{2}$, Case Western Reserve University, Cleveland, OH 44106, USA kal4@ case.edu ${ }^{3}$ NASA Ames Research Center, MS 2694, Moffett Field, CA 94035, USA kai.goebel@nasa.gov ${ }^{4}$ Georgia Institute of Technology, Atlanta, GA 30332, USA gjv@ece.gatech.edu "Exact Nonlinear Filtering and Prediction in Process Model-Based Prognostics", Annual Conference of the Prognostics and Health Management Society, 2009. 1 - 9.

7. Amirah Mohamed Shahiria,* , WahidahHusaina , Nur'aini Abdul Rashida, aSchool of Computer Sciences UniversitiSainsMalayisa 11800 USM, Penang, Malaysia "A Review on Predicting Student's Performance using Data Mining Techniques", The Third Information Systems International Conference, Procedia Computer Science 72 ( 2015 ) $414-422$.

8. Rory P. Bunker a , FadiThabtah b, $\Uparrow$ a Auckland University of Technology, Auckland, New Zealand b Applied Business, Nelson Marlborough Institute of Technology, Auckland, New Zealand "A machine learning framework for sport result prediction", Applied Computing and Informatics (2017). 1 - 7.

9. *Elaf Abu Amrieh ${ }^{1}$, Thair Hamtini ${ }^{2}$ and Ibrahim Aljarah ${ }^{31,2,3}$ Computer Information Systems Department ${ }^{1,2,3}$ The University of Jordan ${ }^{1}$ Ilef.kram@hotmail.com, ${ }^{2}$ thamtini@ju.edu.jo, 3 i.aljarah@ju.edu .jo "Mining Educational Data to Predict Student's academic Performance using Ensemble Methods", International Journal of Database Theory and Application Vol.9, No.8 (2016). Pp. 119-136.

10. HosseinNourkhizMahjoub, Amin Tahmasbi-Sarvestani, HadiKazemi, Yaser P. Fallah "A Learning-based Framework for Two-Dimensional Vehicle Maneuver Prediction Over V2V Networks", 2017 IEEE Cyber Science and Technology Congress (IEEE CyberSciTech).

11. Yunshu Du, Assefaw H. Gebremedhin, and Matthew E. Taylor "Analysis of University Fitness Center Data Uncovers Interesting Patterns, Enables Prediction", IEEE Transactions on Knowledge and Data Engineering. 1-12.

12. R.Subhashini and V. Jawahar Senthil Kumar, "A Framework for Efficient Information Retrieval using NLP Techniques ", Proceedings of the International Conferences on Advances in Communication Network and Computing, CNC 2011, CCIS 142, pp. 391-393, 2011, Springer-Verlag Berlin Heidelberg 2011,ACEEE, Bangalore.

13. R.Subhashini and V. Jawahar Senthil Kumar, "Shallow NLP Techniques for Noun Phrase Extraction", Presented in the International Conference on Trendz in Information Sciences \& Computing (TISC - 2010) in association with Cognizant Technology Solutions and IEEE from 17th to 19th of December, 2010, Sathyabama University, Chennai.

14. Saravanan, M.,Jyothi, V.L.," Enhancement of stress management skills of college students using classification method "Journal of Pharmaceutical Sciences and Research, vol 8(10), 2016 ,pp 1250- 1252.

15. P. Ajitha, Dr. G. Gunasekaran.,'Semantic Based Intuitive Topic Search Engine", International Review of Computers and Software, Praise Worthy Prize, PP 19641970,Vol.9, No.12 Nov 2014. 